\title{
Management of COPD exacerbations: a European Respiratory Society/American Thoracic Society guideline
}

\author{
Jadwiga A. Wedzicha (ERS co-chair) ${ }^{1}$, Marc Miravitlles², John R. Hurst ${ }^{3}$, \\ Peter M.A. Calverley ${ }^{4}$, Richard K. Albert ${ }^{5}$, Antonio Anzueto ${ }^{6}$, Gerard J. Criner ${ }^{7}$ \\ Alberto Papi $\mathbb{1}^{8}$, Klaus F. Rabe ${ }^{9}$, David Rigau ${ }^{10}$, Pawel Sliwinski ${ }^{11}$, Thomy Tonia ${ }^{12}$, \\ Jørgen Vestbo ${ }^{13}$, Kevin C. Wilson ${ }^{14}$ and Jerry A. Krishnan (ATS co-chair) ${ }^{15}$
}

\begin{abstract}
Affiliations: ${ }^{1}$ Airways Disease Section, National Heart and Lung Institute, Imperial College London, London, UK. ${ }^{2}$ Pneumology Dept, Hospital Universitari Vall d'Hebron, CIBER de Enfermedades Respiratorias (CIBERES), Barcelona, Spain. ${ }^{3}$ UCL Respiratory, University College London, London, UK. ${ }^{4}$ Institute of Ageing and Chronic Disease, University of Liverpool, Liverpool, UK. ${ }^{5}$ Dept of Medicine, University of Colorado, Denver, Aurora, CO, USA. ${ }^{6}$ University of Texas Health Science Center and South Texas Veterans Health Care System, San Antonio, TX, USA. ${ }^{7}$ Dept of Thoracic Medicine and Surgery, Lewis Katz School of Medicine at Temple University, Philadelphia, PA, USA. ${ }^{8}$ Respiratory Medicine, Dept of Medical Sciences, University of Ferrara, Ferrara, Italy. ${ }^{9}$ Dept of Internal Medicine, Christian-Albrechts University, Kiel and LungenClinic Grosshansdorf, Airway Research Centre North, German Centre for Lung Research, Grosshansdorf, Germany. ${ }^{10}$ Iberoamerican Cochrane Center, Barcelona, Spain. ${ }^{11}$ 2nd Dept of Respiratory Medicine, Institute of Tuberculosis and Lung Diseases, Warsaw, Poland. 12Institute of Social and Preventive Medicine, University of Bern, Bern, Switzerland. ${ }^{13}$ Division of Infection, Immunity and Respiratory Medicine, University of Manchester, Manchester, UK. ${ }^{14}$ Dept of Medicine, Boston University School of Medicine, Boston, MA, USA. ${ }^{15}$ University of Illinois Hospital and Health Sciences System, Chicago, IL, USA.
\end{abstract}

Correspondence: Marc Miravitlles, Pneumology Dept, Hospital Universitari Vall d'Hebron, Pg. Vall d'Hebron 119-129, Barcelona 08035, Spain. E-mail: mmiravitllesdvhebron.net

@ERSpublications

New guideline on the management of \#COPD exacerbations from @ERStalk and @atscommunity http://ow.ly/Pvtr307YCMu

Cite this article as: Wedzicha JA, Miravitlles M, Hurst JR, et al. Management of COPD exacerbations: a European Respiratory Society/American Thoracic Society guideline. Eur Respir J 2017; 49: 1600791 [https://doi.org/10.1183/13993003.00791-2016]

ABSTRACT This document provides clinical recommendations for treatment of chronic obstructive pulmonary disease (COPD) exacerbations.

Comprehensive evidence syntheses, including meta-analyses, were performed to summarise all available evidence relevant to the Task Force's questions. The evidence was appraised using the Grading of Recommendations, Assessment, Development and Evaluation approach and the results were summarised in evidence profiles. The evidence syntheses were discussed and recommendations formulated by a multidisciplinary Task Force of COPD experts.

After considering the balance of desirable and undesirable consequences, quality of evidence, feasibility, and acceptability of various interventions, the Task Force made: 1) a strong recommendation for noninvasive mechanical ventilation of patients with acute or acute-on-chronic respiratory failure; 2) conditional recommendations for oral corticosteroids in outpatients, oral rather than intravenous corticosteroids in hospitalised patients, antibiotic therapy, home-based management, and the initiation of pulmonary rehabilitation within 3 weeks after hospital discharge; and 3) a conditional recommendation against the initiation of pulmonary rehabilitation during hospitalisation.

The Task Force provided recommendations related to corticosteroid therapy, antibiotic therapy, noninvasive mechanical ventilation, home-based management, and early pulmonary rehabilitation in patients having a COPD exacerbation. These recommendations should be reconsidered as new evidence becomes available. 


\section{Introduction}

The chronic and progressive course of chronic obstructive pulmonary disease (COPD) is often punctuated by "exacerbations", defined clinically as episodes of increasing respiratory symptoms, particularly dyspnoea, cough and sputum production, and increased sputum purulence. COPD exacerbations have a negative impact on the quality of life of patients with COPD $[1,2]$, accelerate disease progression, and can result in hospital admissions and death $[3,4]$.

Evidence-based clinical practice guidelines have been developed by other organisations that recommend inhaled bronchodilator therapy for patients having a COPD exacerbation, as well as supplemental oxygen for hypoxaemic patients [5]. They also make recommendations related to systemic steroids, antibiotic therapy, noninvasive mechanical ventilation (NIV) and home-based management. The purpose of our guidelines is to update the latter recommendations and to address specific questions regarding the treatment of COPD exacerbations that are not answered by existing guidelines. For the following six questions, we employed a systematic review of the literature followed by the Grading of Recommendations Assessment, Development and Evaluation (GRADE) approach to develop treatment recommendations.

1) Should oral corticosteroids be used to treat ambulatory patients who are having a COPD exacerbation?

2) Should antibiotics be used to treat ambulatory patients who are having a COPD exacerbation?

3) Should intravenous or oral corticosteroids be used to treat patients who are hospitalised with a COPD exacerbation?

4) Should NIV be used in patients who are hospitalised with a COPD exacerbation associated with acute or acute-on-chronic respiratory failure?

5) Should a home-based management programme (hospital-at-home) be implemented in patients with COPD exacerbations?

6) Should pulmonary rehabilitation be implemented in patients hospitalised with a COPD exacerbation?

The target audience of this guideline is specialists in respiratory medicine who manage adults with COPD. General internists, primary care physicians, emergency medicine clinicians, other healthcare professionals and policy makers may also benefit from these guidelines. These guidelines provide the basis for rational decisions in the treatment of COPD exacerbations. Clinicians, patients, third-party payers, stakeholders or the courts should never view the recommendations contained in these guidelines as dictates. Though evidence-based guidelines can summarise the best available evidence regarding the effects of an intervention in a given patient population, they cannot take into account all of the unique clinical circumstances that may arise when managing a patient.

While the focus of this guideline is the treatment of COPD exacerbations, the Task Force has also provided a narrative review in the online supplement that answers the following complementary questions. What is the optimal approach to diagnose a COPD exacerbation? What are the conditions to include in the differential diagnosis? What tests are required to assess the severity of a COPD exacerbation? How should a patient be followed during recovery from a COPD exacerbation?

\section{Methods}

\section{Group composition}

The Task Force co-chairs (J.A. Wedzicha and J.A. Krishnan) were selected by the European Respiratory Society (ERS) and American Thoracic Society (ATS). They led all aspects of project management and selected the panellists, which included 11 clinicians with experience in COPD management and research.

This article has supplementary material available from erj.ersjournals.com

Received: April 202016 | Accepted after revision: Nov 152016

The guidelines published by the European Respiratory Society (ERS) incorporate data obtained from a comprehensive and systematic literature review of the most recent studies available at the time. Health professionals are encouraged to take the guidelines into account in their clinical practice. However, the recommendations issued by this guideline may not be appropriate for use in all situations. It is the individual responsibility of health professionals to consult other sources of relevant information, to make appropriate and accurate decisions in consideration of each patient's health condition and in consultation with that patient and the patient's caregiver where appropriate and/or necessary, and to verify rules and regulations applicable to drugs and devices at the time of prescription.

This document was endorsed by the ERS Executive Committee and approved by the ATS Board of Directors in December 2016

Conflict of interest: D. Rigau and T. Tonia act as methodologists for the European Respiratory Society. All other disclosures can be found alongside this article at erj.ersjournals.com 
In addition, there were two methodologists ( $\mathrm{T}$. Tonia and $\mathrm{D}$. Rigau) and a clinician-methodologist (K.C. Wilson). The lead methodologist (T. Tonia) identified and collected the evidence, performed the evidence syntheses, constructed the evidence profiles, and ensured that all the methodological requirements were met, with assistance from the other methodologists. The co-chairs and panellists discussed the evidence and formulated the recommendations; the methodologists did not participate in the development of recommendations. All panel members were required to disclose their conflicts of interest. At least $50 \%$ of the co-chairs and $50 \%$ of the panel were required to be free from conflicts of interest. Individuals with potential conflicts of interest took part in the discussions about the evidence but did not participate in the formulation of recommendations.

\section{Formulation of questions}

Task Force members compiled a list of issues that they considered important and relevant to the treatment of COPD exacerbations. The questions were rephrased by the lead methodologist using the PICO (Population, Intervention, Comparator and Outcomes) format [6]. Discussion and consensus among the co-chairs and panellists was used to identify the six questions that would be addressed in the guideline.

\section{Rating the importance of outcomes}

After choosing the questions, the Task Force identified outcomes that they considered relevant to each question. They rated the importance of each outcome using a scale from 1 to 9 (a rating of 1-3 was assigned to outcomes of low importance for decision-making, 4-6 to outcomes important for decision-making and 7-9 to outcomes critically important for decision-making). A teleconference was convened during which the ratings were discussed and some additional outcomes were rated. At the conclusion of the teleconference, all outcomes were categorised as "not important", "important" or "critical" for decision-making.

\section{Literature searches}

Our literature searches used the National Institute of Health and Clinical Excellence (NICE) guidelines as a starting point [5,7]. For questions that were addressed in the 2004 NICE guidelines, we conducted literature searches in Medline, Embase and the Cochrane Database of Systematic Reviews beginning in 2003. For questions that were addressed in the 2010 NICE guidelines, we conducted literature searches in the same databases beginning in 2009. Initial searches were conducted in January 2012, and then updated in June 2012, February 2013 and September 2015. We used the same or similar search strategies as those used by NICE. To search Embase and Medline, we searched only the English-speaking literature using the search strategy shown in the online supplement, whereas to search the Cochrane Database of Systematic Reviews, we used the search term "chronic obstructive pulmonary disease".

\section{Study selection}

The lead methodologist screened the titles and abstracts of the retrieved studies, and excluded studies on the basis of the pre-defined study selection criteria shown in the online supplement. For those studies that could not be excluded by the title and abstract, we obtained the full text of the articles and then included or excluded the studies on the basis of our full text review. In cases of uncertainty, the opinions of the co-chairs and panellists were obtained and decisions were reached by discussion and consensus. We also screened the reference lists from recent and systematic reviews to ensure that our literature review had not missed relevant studies.

\section{Evidence synthesis}

Study characteristics, types of participants, interventions, the outcomes measured and results were extracted from each study. If the data were amenable to pooling, effects were estimated via meta-analysis using Review Manager (version 5.3; The Nordic Cochrane Centre, Copenhagen, Denmark). For the meta-analyses, the random effects model was utilised unless otherwise specified. Dichotomous outcomes were reported as relative risks and continuous outcomes were reported as mean differences unless otherwise specified. The lead methodologist appraised the quality of evidence using the GRADE approach [8].

The lead methodologist used GRADEpro (McMaster University, Hamilton, ON, Canada) to develop evidence profiles that summarised the findings for each outcome and the rationale for the quality of evidence appraisal. Thresholds for clinically important changes (used to judge imprecision) included the following relative risk reductions: mortality $15 \%$, exacerbations $20 \%$, hospitalisations $20 \%$, treatment failure $20 \%$ and adverse events 15\%. They also included the following absolute reductions: a St George's Respiratory Questionnaire score change of 4 points and a forced expiratory volume in $1 \mathrm{~s}$ (FEV1) change of $100 \mathrm{~mL}$. The thresholds for clinically important relative risk reductions were based upon the Task Force's collective clinical experience and, for consistency, were chosen to be similar to the thresholds used 
to develop the NICE guidelines on COPD [7]. The thresholds for clinically important absolute risk reductions were based upon published literature [9].

\section{Formulating and grading recommendations}

The evidence profiles were sent to the Task Force members for review. Using an iterative consensus process conducted face to face, via teleconference and via email, recommendations were formulated on the basis of the following considerations: the balance of desirable (benefits) and undesirable consequences (burden, adverse effects and cost) of the intervention, the quality of evidence, patient values and preferences, and feasibility [10].

A strong recommendation was made for an intervention when the panel was certain that the desirable consequences of the intervention outweighed the undesirable consequences, just as a strong recommendation would have been made against an intervention if the panel was certain that the undesirable consequences of the intervention outweigh the desirable consequences. A strong recommendation indicates that most well-informed patients would choose to have or not to have the intervention.

A conditional recommendation was made for an intervention when the panel was uncertain that the desirable consequences of the intervention outweighed the undesirable consequences, just as a conditional recommendation would have been made against an intervention if the panel was uncertain that the undesirable consequences of the intervention outweigh the desirable consequences. Reasons for uncertainty included low or very low quality of evidence, the desirable and undesirable consequences being finely balanced, or the underlying values and preferences playing an important role. A conditional recommendation indicates that well-informed patients may make different choices regarding whether to have or not have the intervention.

\section{Manuscript preparation}

The initial draft of the manuscript was prepared by the co-chairs, methodologists and one panellist (M. Miravitlles). The panel members wrote the content for the online supplement, which was collated and edited by the co-chairs. Both the manuscript and the online supplement were reviewed, edited and approved by all panel members prior to submission.

\section{Results}

Should oral corticosteroids be used to treat patients whose COPD exacerbation is mild enough to be treated as an outpatient (i.e. ambulatory patients)?

Summary of the evidence

We identified three relevant systematic reviews [11-13], which identified two trials that evaluated the effects of oral corticosteroids in ambulatory patients having a COPD exacerbation $[14,15]$. Our own systematic review identified a third clinical trial [16]. These three trials in a total of 204 patients informed the Task Force's judgments [14-16]. The first trial enrolled 27 ambulatory patients who were having a COPD exacerbation, defined as subjective worsening of baseline cough or dyspnoea for more than $24 \mathrm{~h}$, requiring a hospital visit, and at least one of the following: a $25 \%$ increase in $\beta$-agonist use, increased sputum production or increased sputum purulence [15]. The patients were randomly assigned to receive a tapering dose of prednisone or placebo for 9 days and followed for 14 days following the completion of the tapering dose. The second trial enrolled 147 patients who were being discharged from the emergency room after being seen for a COPD exacerbation, defined as having at least two of the following: a recent increase in breathlessness, sputum volume or sputum purulence [14]. The patients were randomly assigned to receive either $40 \mathrm{mg}$ of oral prednisone or placebo for 10 days and then followed for 30 days from the initiation of treatment. The most recent trial randomly assigned 30 ambulatory patients who were having a COPD exacerbation to receive $30 \mathrm{mg}$ of oral prednisolone or placebo for 14 days and then followed during the treatment course only [16].

The Task Force identified four outcomes a priori as critical to guiding treatment recommendations: treatment failure (composite of unscheduled visit to the physician, return to the emergency department because of worsening respiratory symptoms, hospitalisation or unmasking of study medication due to worsening respiratory symptoms), hospital admissions, mortality and time to next COPD exacerbation. Change in quality of life and serious adverse events were considered "important" outcomes to guide treatment recommendations.

When the data were pooled via meta-analysis (see evidence profile 1 in the online supplementary material), oral corticosteroids caused a trend toward fewer hospital admissions (7.9\% versus 17\%; relative risk (RR) $0.49,95 \%$ CI $0.23-1.06)$. There was no significant difference in treatment failure (26.5\% versus 42.4\%; RR $0.69,95 \%$ CI $0.22-2.19$ ) or mortality (1.1\% versus $1.1 \%$; RR 0.99 , 95\% CI $0.06-15.48$ ). The effect on treatment failure would be clinically important if real, but there were too few events to confirm or exclude the effect and the analysis was limited by severe heterogeneity of uncertain cause, as sensitivity analyses failed eliminate the heterogeneity. Data regarding length of hospital stay or time to next 
exacerbation were not reported in the three studies. Patients who received oral corticosteroids had better lung function, measured as the FEV1 (mean difference $0.16 \mathrm{~L}$ higher, 95\% CI 0.04-0.28 L higher) but no significant difference in quality of life measured by the Chronic Respiratory Questionnaire score (mean difference 0.38 higher, $95 \%$ CI 0.09 lower to 0.85 higher) or serious adverse effects $(2.2 \%$ versus $1.1 \%$; RR $1.97,95 \%$ CI $0.18-21.29)$.

\section{Benefits}

Oral corticosteroids improved lung function in ambulatory patients having a COPD exacerbation. There was also a trend toward fewer hospitalisations.

\section{Harms}

Various adverse effects were reported in the studies, including seizures, insomnia, weight gain, anxiety, depressive symptoms and hyperglycaemia. However, it is unclear whether the methods used to assess harms were similar across the studies and there were too few serious adverse events reported to adequately evaluate the difference in the risk of harms with oral corticosteroids versus placebo in patients with COPD exacerbations treated in the ambulatory setting.

\section{Other considerations}

There was no information in any of the trials regarding the time to next exacerbation and inadequate information to have confidence regarding the effects of systemic corticosteroids on several outcomes considered critical or important to decision making (hospitalisation, mortality and serious adverse events).

\section{Conclusions and research needs}

A course of oral corticosteroids for 9-14 days in outpatients with COPD exacerbations improves lung function and causes a trend toward fewer hospitalisations. No effect on treatment failure, mortality or adverse effects has been demonstrated, although there were too few events in the trials to definitively confirm or exclude an effect on any of these outcomes. The Task Force judged that the benefits of oral corticosteroids probably outweigh the adverse effects, burdens and costs, but was uncertain due to its very low confidence in the accuracy of the estimated effects.

Phenotypic identification of responders to oral corticosteroids is an area of research that should be explored. There are some data suggesting that patients with an elevated blood eosinophil count will respond more to oral corticosteroids than patients with a low blood eosinophil count. One randomised trial found that patients whose blood eosinophil count was $\geqslant 2 \%$ had greater improvement in their health-related quality of life and faster recovery after receiving oral corticosteroids compared to placebo. In contrast, in patients whose blood eosinophil count was $<2 \%$ there was a significantly greater improvement in health-related quality of life in patients receiving placebo [17]. Another study pooled data from three randomised trials of patients with a COPD exacerbation and found that systemic steroid-treated patients with a blood eosinophil count $\geqslant 2 \%$ have a treatment failure rate of only $11 \%$, compared with a treatment failure rate of $66 \%$ among those in placebo arm. However, among patients with blood eosinophils $<2 \%$, the rate of failure was $26 \%$ with prednisone and only $20 \%$ with placebo [18]. Larger randomised controlled trials with stratification by blood eosinophil count are needed. Several studies suggest that an even shorter duration of systemic corticosteroid treatment (e.g. 3 days [19], 5 days [20] or 7 days [21]) may be as effective as longer courses in hospitalised patients with exacerbations of COPD; similar studies need to be performed in ambulatory patients. Finally, effectiveness studies conducted in real-life situations should be conducted to confirm the findings of efficacy trials.

What others are saying

The 2010 NICE guidelines [5] concluded that, in the absence of significant contraindications, oral corticosteroids should be used in conjunction with other therapies in all patients admitted to hospital with an exacerbation of COPD and considered in patients in the community who have an exacerbation with a significant increase in breathlessness that interferes with daily activities. The 2014 Global Initiative for Chronic Obstructive Lung Disease (GOLD) strategy document [22] concluded that "systemic corticosteroids are beneficial in the management of COPD. They shorten recovery time, improve lung function and hypoxemia, and may reduce the risk of early relapse, treatment failure, and length of hospital stay. A dose of $30-40 \mathrm{mg}$ prednisone per day for 5 days is recommended".

\section{ERS/ATS recommendation}

For ambulatory patients with an exacerbation of COPD, we suggest a short course ( $\leqslant 14$ days) of oral corticosteroids (conditional recommendation, very low quality of evidence). 
Remarks

The Task Force defines a short course of oral corticosteroids as $\leqslant 14$ days.

Values and preferences

This recommendation places a high value on a reduction in treatment failure and a lower value on the uncertainty regarding the potential for adverse events.

\section{Should antibiotics be administered to ambulatory patients who are having a COPD exacerbation?}

Summary of the evidence

We identified three systematic reviews [23-25], which included four trials that evaluated antibiotic therapy in ambulatory patients with COPD exacerbations [26-29]. Our own systematic review identified an additional relevant trial that was not included in the published systematic reviews [30]. We pooled two of the five trials $[26,30]$ that enrolled a total of 483 participants via meta-analysis to inform the Task Force's judgments (evidence profile 2 in the supplementary material). The remaining three trials were excluded because the diagnosis of COPD was inadequately established among patients enrolled [27], data on treatment failure were measured on day 5 [29] and the panel believed that 5 days are not enough to judge whether an exacerbation has resolved [31], and publication was as an abstract only [28].

The Task Force identified a priori six outcomes as critical to guiding treatment recommendations: treatment failure (composite of death, no resolution or deterioration), adverse events, time to next COPD exacerbation, hospitalisation, length of hospital stay and death.

Among the trials that were pooled, one randomly assigned 310 ambulatory patients who were having a COPD exacerbation to receive placebo or amoxicillin/clavulanate for 8 days [30], while the other randomly assigned 116 similar patients to receive placebo or any one of the following for 7-10 days: trimethoprim/ sulfamethoxazole, amoxicillin or doxycycline [26]. Antibiotic therapy decreased treatment failure $(27.9 \%$ versus $42.2 \%$; RR 0.67 , 95\% CI 0.51-0.87); this effect was driven entirely by lack of resolution and deterioration, since no deaths were reported. It also prolonged the time to the next exacerbation (difference of medians 73 days, $\mathrm{p}=0.015$ ). There was a trend toward more adverse events among patients who received antibiotic therapy (14.6\% versus $7.9 \%$; RR $1.84,95 \%$ CI $0.95-3.57$ ), although most of the adverse events were described as mild. Data regarding hospitalisation, length of hospital stay and death were not reported.

\section{Benefits}

Antibiotic therapy reduced the risk of treatment failure and increased the time between COPD exacerbations.

Harms

Patients who received antibiotic therapy had a trend toward more adverse events, most of which were mild gastrointestinal side effects (e.g. diarrhoea).

\section{Other considerations}

In this evaluation of ambulatory exacerbations, there was no information in either trial about several outcomes of interest to the Task Force; specifically, the hospital admission rate, length of hospital stay and mortality.

Conclusions and research needs

The use of antibiotics in ambulatory patients with exacerbations of COPD reduces the treatment failure rate and increases the time to the next exacerbation. However, the majority of patients avoided treatment failure even in the placebo group (58\%), suggesting that not all exacerbations require treatment with antibiotics. Effectiveness studies should be conducted in real-life situations to confirm the findings of efficacy trials. Identifying biomarkers of bacterial infection may allow the patient population that definitively requires antibiotic treatment to be more precisely selected [32]. Additional research is needed to identify patients in whom antibiotic therapy is needed.

What others are saying

The 2010 NICE guidelines [5] advise that antibiotics should be used to treat exacerbations of COPD associated with purulent sputum. However, the recommendation is not specific for ambulatory patients with COPD exacerbations. The 2014 GOLD strategy document [22] states that antibiotics should be given to patients with COPD exacerbations who fulfil certain criteria; again, the recommendation is not specific to ambulatory patients having an exacerbation of COPD. 


\section{ERS/ATS recommendation}

For ambulatory patients having a COPD exacerbation, we suggest the administration of antibiotics (conditional recommendation, moderate quality of evidence). Antibiotic selection should be based upon local sensitivity patterns.

\section{Remarks}

Studies suggest that episodes that present with purulent sputum are most likely to benefit from antibiotic treatment; however, there may be other considerations (e.g. disease severity) when deciding whether or not to prescribe an antibiotic [22].

\section{Values and preferences}

This recommendation places a high value on a reduction in treatment failure and extending the time between exacerbations, and a lower value on avoiding adverse events.

\section{Should intravenous or oral corticosteroids be used to treat patients who are hospitalised with a} COPD exacerbation?

\section{Summary of the evidence}

There is evidence supporting the use of systemic corticosteroids in patients with severe exacerbations of COPD treated in the hospital $[5,22]$. However, high-dose intravenous corticosteroids for admitted patients with severe exacerbations may not have a higher efficacy than oral corticosteroids and can potentially be associated with a higher risk of adverse events; therefore, we searched for evidence comparing both routes of administration of corticosteroids in this population of patients.

We did not identify any systematic reviews comparing intravenous corticosteroids with oral corticosteroids in hospitalised patients with COPD exacerbations. Our own systematic review identified two trials in a total of 250 patients hospitalised with a COPD exacerbation [33, 34]. One trial randomly assigned 210 hospitalised patients with COPD exacerbations to receive either $60 \mathrm{mg}$ of intravenous prednisolone plus oral placebo or $60 \mathrm{mg}$ of oral prednisolone plus intravenous placebo for 5 days [33]. Both groups received an oral prednisolone taper following the 5 days of full-dose therapy (total duration 10 days). The other trial randomly assigned 40 patients to receive either $32 \mathrm{mg}$ per day of oral methylprednisolone for 7 days or $1 \mathrm{mg} \cdot \mathrm{kg}^{-1}$ per day of intravenous methylprednisolone for 4 days followed by $0.5 \mathrm{mg} \cdot \mathrm{kg}^{-1}$ per day of intravenous methylprednisolone for 3 days (total duration 10 days) [34].

The Task Force identified a priori five outcomes as "critical" to guiding treatment recommendations: treatment failure (composite of death, admission to the intensive care unit (ICU), readmission to the ICU due to COPD or intensification of pharmacological therapy), mortality, readmission to the hospital, length of hospital stay and time next COPD exacerbation. Adverse events were considered important outcomes to guide treatment recommendations.

When the trial results were pooled (evidence profile 3 in the supplementary material), there were no significant differences in treatment failure $53.5 \%$ for intravenous versus $49.6 \%$ for oral corticosteroids; RR $1.09,95 \%$ CI $0.87-1.37$ ), mortality (5.5\% for intravenous versus $1.7 \%$ for oral corticosteroids; RR $2.78,95 \%$ CI $0.67-11.51$ ), hospital readmissions (14.2\% for intravenous versus $12.4 \%$ for oral corticosteroids; RR $1.13,95 \%$ CI $0.60-2.13$ ), or length of hospital stay (mean difference of 0.71 more days with intravenous steroids than oral steroids, $95 \%$ CI ranged from 1.35 fewer days to 2.78 more days). Data regarding time to next exacerbation were not reported in the studies.

One trial demonstrated an increased risk of mild adverse effects in the intravenous corticosteroids group (70\% versus $20 \%$; RR 3.50, 95\% CI 1.39-8.8) [34], which were easily treated with appropriate medications. Of note, the intravenous arm used a higher dose of corticosteroids than the oral arm; therefore, it is unknown whether the increased incidence of adverse effects was due to the route of administration or the dose. Neither trial reported any serious adverse effects.

\section{Benefits}

Among outcomes that are known to be improved by corticosteroids therapy (i.e. reduced treatment failure), there were no differences between oral and intravenous therapy.

Harms

Only one study (which enrolled a total of 40 participants) reported the frequency of adverse events, which were numerically higher in the group treated with intravenous corticosteroids than with oral corticosteroids (e.g. 11 versus four developed hyperglycaemia and three versus none had worsening of hypertension, respectively) [34]. However, these assessments were not performed masked to treatment assignment and there were too few events to make definitive conclusions about the relative risk of adverse 
events with either therapy. A large observational study of 80000 non-ICU patients hospitalised with COPD exacerbations suggests that $>90 \%$ of practitioners in the USA favour use of intravenous over oral corticosteroids in this population [35]. Interestingly, patients in this study treated with intravenous corticosteroids had a longer length of stay and higher cost compared to those treated with oral corticosteroids, without clear evidence of benefit (assessed using the composite outcome of death, need for mechanical ventilation or 30-day readmission) [36].

\section{Other considerations}

There was no information in either trial about one of the outcomes of interest to the Task Force: the time to next exacerbation. There was a serious risk of bias due to lack of blinding for most outcomes, and the number of events and patients were small for all outcomes; these features decreased the panel's confidence in the estimated effects.

Conclusions and research needs

Treatment failure, hospital readmissions and length of hospital stay are not significantly different among patients who receive oral or intravenous corticosteroids; however, the results indicate that intravenous therapy might increase the risk of adverse effects. No effect on mortality has been shown, although there were too few deaths in the trials to definitively confirm or exclude an effect on mortality. Since the studies did not employ a noninferiority design and the confidence intervals indicated imprecision for both benefits and harms, we cannot conclude that both intravenous and oral corticosteroids confer similar benefits and harms. There is therefore insufficient evidence to support one method of administration over the other. An adequately powered noninferiority trial comparing the relative harms and benefits of intravenous versus oral corticosteroids in this population is needed, particularly given the potential for increasing the length of stay and healthcare costs with intravenous therapy, as observed in the observational study.

What others are saying

The 2010 NICE guidelines [5] did not compare oral and intravenous corticosteroids. The 2014 GOLD strategy document [22] says that the oral prednisolone is preferable.

\section{ERS/ATS recommendation}

For patients who are hospitalised due to a COPD exacerbation, we suggest the administration of oral corticosteroids rather than intravenous corticosteroids if gastrointestinal access and function are intact (conditional recommendation, low quality of evidence).

\section{Remarks}

Intravenous corticosteroids should be administered to patients who are unable to tolerate oral corticosteroids. Foregoing corticosteroid therapy in patients who cannot tolerate oral therapy is not an option due to the benefits of corticosteroid therapy.

\section{Values and preferences}

This recommendation places a high value on the simplicity of providing oral compared to intravenous corticosteroids and the potential to reduce healthcare expenditures with oral therapy, rather than convincing evidence about benefits or harms supporting one form of administration over the other.

\section{Should NIV be used in patients who are hospitalised with a COPD exacerbation associated with} acute or acute-on-chronic respiratory failure?

Summary of the evidence

We identified a systematic review [37] that included 14 randomised trials that evaluated the effects of NIV on patients with acute respiratory failure due to a COPD exacerbation [48-51]. Our own systematic review identified an additional seven relevant trials [52-58]. These 21 trials formed the evidence base that was used to inform the Task Force's judgments. Many of the trials excluded patients with any of the following: inability to cooperate, protect the airway or clear secretions; severely impaired consciousness; facial deformity; high aspiration risk; or recent oesophageal stenosis.

The Task Force identified a priori five outcomes as critical to guiding treatment recommendations: death, intubation, length of hospital stay, length of ICU stay and nosocomial pneumonia. Complications of treatment (e.g. aspiration or barotrauma) and $\mathrm{pH} 1 \mathrm{~h}$ after intervention were considered important outcomes.

All of the trials enrolled hospitalised patients with respiratory failure due to a COPD exacerbation. In the overwhelming majority of the studies, the patients had confirmed acute or acute-on-chronic hypercapnic respiratory failure; a few of the studies did not specify that the respiratory failure was hypercapnic. Most the trials compared usual care plus NIV to usual care alone, although a few assigned patients to usual care 
plus NIV or usual care plus sham NIV. Due to the nature of the intervention, most of the trials were not blinded to the patients, caregivers or assessors.

When the trials were pooled via meta-analysis (evidence profile 4 in the supplementary material), patients who received NIV had a lower mortality rate (7.1\% versus $13.9 \%$; RR 0.54 , 95\% CI $0.38-0.76$ ), were less likely to require intubation ( $12 \%$ versus $30.6 \%$; RR 0.43 , 95\% CI $0.35-0.53$ ), had a shorter length of hospital stay (mean difference 2.88 days fewer, 95\% CI 1.17-4.59 days fewer) and ICU stay (mean difference 4.99 days fewer, 95\% CI 0-9.99 days fewer) and had fewer complications of treatment (15.7\% versus $42 \%$; RR 0.39 , 95\% CI 0.26-0.59). There was no difference in the $\mathrm{pH}$ after $1 \mathrm{~h}$ (mean difference 0.02 , 95\% CI 0.01-0.06). When we repeated the analyses using only the studies that had confirmed acute or acute-on-chronic hypercapnic respiratory failure, the results were essentially the same.

Benefits

NIV reduced the need for intubation, mortality, complications of therapy, and length of both hospital stay and ICU stay in patients with acute or acute-on-chronic respiratory failure due to a COPD exacerbation.

Harms

There were no reports of adverse consequences; to the contrary, complications of therapy were reduced in patients who received NIV.

\section{Other considerations}

Most of the trials had a serious risk of bias due to uncertain allocation concealment and lack of blinding. For some outcomes, the estimated effects were inconsistent across studies or the number of events and patients were small, diminishing confidence in the estimated effects. Similarly, one of the outcomes of interest, the rate of nosocomial pneumonia, could not be assessed because the data were either not reported or incompletely reported. These considerations contributed to grading the quality of evidence as low.

\section{Conclusions and research needs}

Use of NIV in patients with acute or acute-on-chronic respiratory failure due to a COPD exacerbation reduces the need for intubation, mortality, complications of therapy, length of hospital stay and length of ICU stay. Future research will determine strategies for optimising the delivery of NIV, including the optimal technique and interface type selection. We need studies to address how to titrate and wean patients from NIV ventilation, and how to better determine which physiological effects should be expected during the application of NIV that predict treatment success or failure. The efficacy of home NIV in patients following a COPD-related hospitalisation when NIV was utilised to treat acute-on-chronic respiratory failure is also an area that requires additional study. Recent data have reported conflicting outcomes regarding home NIV in the severe COPD outpatient population [59-62]. Effectiveness studies should be conducted in real-life situations to confirm the findings of efficacy trials. Other research opportunities are related to decision-making about whether or when to intubate or not, as well as the use of NIV by healthcare providers, patients and family members.

What others are saying

The 2010 NICE guidelines [5] did not discuss the use of NIV in COPD exacerbations. In the 2004 NICE guidelines, however, it was stated that NIV should be used as the treatment of choice for persistent hypercapnic ventilatory failure during exacerbations despite optimal medical therapy. The 2014 GOLD strategy document [22] states that, in patients with acute respiratory failure due to a COPD exacerbation, NIV improves respiratory acidosis and decreases the intubation rate, mortality, respiratory rate, severity of breathlessness, complications (e.g. ventilator-associated pneumonia) and length of hospital stay. They recommend the use of NIV in patients with 1) respiratory acidosis or 2) severe dyspnoea with clinical signs suggestive of respiratory muscle fatigue, increased work of breathing, or both, such as use of respiratory accessory muscles, paradoxical motion of the abdomen or retraction of the intercostal spaces.

\section{ERS/ATS recommendation}

For hospitalised patients with acute or acute-on-chronic hypercapnic respiratory failure due to a COPD exacerbation, we recommend the use of NIV (strong recommendation, low quality of evidence).

Remarks

The strong recommendation despite the panel's low confidence in the estimated effects reflects the panel's consensus opinion that the overwhelming majority of patients would want NIV given the possibility of one or more important clinical benefits with minimal risk of harm. Many of the trials excluded patients 
with any of the following: inability to cooperate, protect the airway or clear secretions; severely impaired consciousness; facial deformity; high aspiration risk; or recent oesophageal stenosis.

\section{Values and preferences}

This recommendation places a high value on reducing mortality and the need for invasive mechanical ventilation, and lower value on the burdens associated with NIV.

\section{Should a home-based management programme (hospital-at-home) be implemented in patients with COPD exacerbations? \\ Summary of the evidence}

A home-based management programme involving nurses and potentially other healthcare professionals (e.g. physicians, social workers and physical therapists), also known as "hospital-at-home", offers the option of an early assisted hospital discharge or an alternative to hospitalisation in patients presenting to the emergency department with a COPD exacerbation. Clinical trials have compared home-based management to usual care in patients with COPD exacerbations who meet other additional eligibility criteria (e.g. no impairment of consciousness, decompensated heart failure or other acute condition, or need for mechanical ventilation). We found a systematic review [63] that included eight relevant trials [64-71]. Our own systematic review identified one additional trial [72]. These nine trials formed the evidence base that was used to inform the Task Force's judgment. All of the trials enrolled patients who presented with COPD exacerbations; five trials evaluated hospital admission versus discharge to a hospital-at-home from the emergency department [65-67, $69,70]$, three trials assessed ongoing hospital admission versus discharge to a hospital-at-home following an initial hospitalisation $[64,71,72]$ and in one trial, the setting of the discharge could not be determined [68]. Four trials were conducted in the UK [64, 65, 69, 71], four trials were conducted in other European countries $[66,68,70,72]$ and one trial was conducted in Australia [67].

The Task Force identified a priori three outcomes as critical to guiding treatment recommendations: death, hospital readmission and time to first readmission. Hospital-acquired infections and quality of life were considered important outcomes.

When the trials were pooled via meta-analysis (evidence profile 5 in the supplementary material), home-based management reduced hospital readmissions (26.8\% versus $34.2 \%$; RR 0.78 , 95\% CI $0.62-0.99$ ) and was associated with a trend towards lower mortality (5.6\% versus $8.5 \%$; RR $0.66,95 \%$ CI $0.41-1.05$ ). There was no difference in the time to first readmission (mean difference of 8 days longer among patients in the home-based management group, 95\% CI 19.7 days longer to 3.7 days shorter). No data were reported on hospital-acquired infections or quality of life.

The Task Force raised the possibility that a home-based management may have different effects among patients who are discharged from the emergency department compared to patients who are discharged following an initial hospitalisation. To address these concerns, a post hoc stratified analysis was performed (evidence profile 5); the results of these analyses did not provide convincing evidence to indicate differential effects among patients discharged from different locations or to exclude the possibility of heterogeneity of treatment effects.

\section{Benefits}

Utilisation of a home-based management model reduced the number of hospital readmissions and, possibly, mortality in patients with COPD exacerbations.

\section{Harms}

Adverse events were not an outcome reported in any of the included trials; therefore, there exists no data regarding the potential harms of the home-based management model.

\section{Other considerations}

For most of the outcomes, the number of events and patients in the trials were small, diminishing confidence in the estimated effects. There was no information reported for one outcome of interest to the Task Force, the rate of hospital-acquired infections. In addition, there was insufficient information to draw conclusions regarding another outcome of interest, quality of life (i.e. among the three trials that reported quality of life, one did not provide standard deviations, another only provided St George's Respiratory Questionnaire scores for a subgroup of participants and a third measured generic health-related quality of life using the EuroQoL-5D scale). Moreover, the eligibility criteria varied across studies and the capacity of health systems to deliver home-based care for this population may vary. There is also a large geographical variability in their availability. Studies are also needed to identify the components of home-based COPD care required for benefit and how such requirements may vary based on the variable contexts in which patients live. 
Although not pre-specified by the Task Force as outcomes of interest, it is worth noting that four trials reported costs, and three reported patient and provider satisfaction. Among the trials that evaluated costs, two found lower costs for hospital-at-home programmes [67, 70], one found a trend toward lower costs [66] and one found no difference [73]. Among the three trials that evaluated patient and provider satisfaction, all reported no differences $[69,70,74]$. While no differences in overall satisfaction were found, the majority of patients indicated that they would prefer home treatment if they were allowed to choose.

\section{Conclusions and research needs}

The home-based management programme model in patients with a COPD exacerbation reduces hospital admissions, making it a safe and effective way of discharging patients with additional home-based support in appropriately selected patients. This may increase the availability of hospital beds and reduce pressure on clinicians to discharge patients whose readiness is uncertain. The home-based model might also reduce mortality; however, there were too few deaths in the trials to definitively confirm or exclude an effect.

One of the major research needs for home-based management is the development of algorithms to screen patients to determine which are or are not appropriate for home-based care. Some studies suggest that home treatment of COPD exacerbations should be considered in all patients unless there are mental status changes, confusion, hypercarbia, refractory hypoxaemia, serious comorbid conditions or inadequate social support. However, these criteria need to be evaluated prospectively to define the most appropriate selection criteria. The feasibility of home-based administration of medications for COPD exacerbations (i.e. systemic corticosteroids, antibiotics, nebulised bronchodilators and supplemental oxygen) may vary by patient characteristics (e.g. ability to carry out activities of daily living and level of social support), or by the capacity of the health system or home health agency. Studies are needed to define the patient selection criteria and key elements of the home-based programme (e.g. nurse or inter-professional teams that include a physician, respiratory therapist or social worker; treatment plan at home; criteria for treatment failure at home; and need for hospitalisation). Finally, studies are needed to prospectively evaluate the potential for heterogeneity of treatment effects according to whether the home-based management programme is intended to avoid a hospitalisation or to facilitate early discharge from the hospital to home. Many of these studies may be best conducted as effectiveness studies in real-life situations; at a minimum, effectiveness studies should be conducted to confirm the findings of efficacy trials.

What others are saying

The 2010 NICE guidelines [5] did not include a section on home-based management of patients with COPD exacerbations, but referred to it briefly as something that respiratory nurse specialists might be involved in. The 2014 GOLD strategy document [22] stated that "hospital at home represents an effective and practical alternative to hospitalisation in selected patients with exacerbations of COPD without acidotic respiratory failure. However, the exact criteria for this approach as opposed to hospital treatment remain uncertain and will vary by healthcare setting. Treatment recommendations are the same for hospitalised patients".

\section{ERS/ATS recommendation}

For patients with a COPD exacerbation who present to the emergency department or hospital, we suggest a home-based management programme (hospital-at-home; conditional recommendation, moderate quality of evidence).

\section{Remarks}

Appropriately selected patients may include those who do not have acute or acute-on-chronic ventilatory respiratory failure, respiratory distress, hypoxaemia requiring high-flow supplemental oxygen, an impaired level of consciousness, cor pulmonale, a need for full-time nursing care, other reasons for hospitalisation (e.g. myocardial ischaemia), housing or food insecurity, poor social support, or active substance abuse.

\section{Values and preferences}

This recommendation places a high value on reducing hospital readmissions, improving patient safety and potentially also decreasing mortality, and a lower value on the burdens of caring for acutely ill patients at home.

\section{Should pulmonary rehabilitation be implemented in patients hospitalised with a COPD exacerbation? \\ Summary of the evidence}

We identified a systematic review [75] that included nine trials that randomly assigned hospitalised patients with COPD exacerbations to early pulmonary rehabilitation plus usual care or usual care alone [76-84]. The pulmonary rehabilitation programmes all included physical exercise that was initiated within 3 weeks of initiating treatment for a COPD exacerbation treatment; in five trials, pulmonary rehabilitation was initiated 
during the hospitalisation $[76,78,79,82,84]$ and, in three trials, pulmonary rehabilitation was initiated following discharge $[80,81,83]$. We excluded one of the trials because the patients had already completed a pulmonary rehabilitation programme in the past and the trial assessed a repeat programme [77].

Our own systematic review identified five additional relevant randomised trials [85-89], with two studies enrolling hospitalised patients $[88,89]$ and three studies enrolling patients up to 8 weeks after hospital discharge [85-87]. Each trial implemented pulmonary rehabilitation differently: health education and exercise training, beginning within 2 months following hospital discharge [85]; training in breathing techniques and physical exercise, beginning 2-3 weeks after hospital discharge [86]; strength and aerobic exercise training, chest physiotherapy for secretion drainage, breathing retraining, nutrition and psychosocial support, beginning within 2 weeks after discharge [87]; twice-daily exercise training of varying intensity, initiated during hospitalisation [88]; and progressive strength and aerobic exercise, initiated within $48 \mathrm{~h}$ of admission [89].

These 13 trials formed the evidence base used to inform the Task Force's decisions. The Task Force identified a priori three outcomes as critical to guiding the formulation of treatment recommendations: death, hospital readmission and quality of life. Exercise capacity was considered an important outcome.

Pooling the trials via meta-analysis (evidence table 6) suggested that pulmonary rehabilitation following admission for an exacerbation may have reduced hospital readmissions (45.0\% versus 50.8\%; RR 0.65, 95\% CI 0.42-1.00), improved quality of life as measured by a change in the St George's Respiratory Questionnaire score (mean difference $-11.75,95 \%$ CI -19.76 to -3.75 ) and improved exercise capacity as measured by the 6-min walking test (mean difference $+88.89 \mathrm{~m}, 95 \%$ CI $+26.67 \mathrm{~m}$ to $+151.11 \mathrm{~m}$ ). However, these estimates were uncertain due to inconsistent results for across trials $\left(\mathrm{I}^{2}=69 \%\right.$ for hospital readmissions, $\mathrm{I}^{2}=70 \%$ for quality of life and $\mathrm{I}^{2}=97 \%$ for exercise capacity). With respect to mortality, we excluded one trial from the mortality analysis because the panel decided that its measurement of deaths in the ICU was potentially misleading [82]; when the remaining trials were pooled, there was no significant difference among those who did or did not receive pulmonary rehabilitation (19.6\% versus $14.1 \%$; RR 1.44 , 95\% CI $0.97-2.13 ; \mathrm{I}^{2}=0 \%$ for mortality).

The panel hypothesised that differences in the timing of the initiation of pulmonary rehabilitation may have been the cause of the inconsistent results across trials. To test this hypothesis, a post hoc stratified analysis was performed. Patients who initiated pulmonary rehabilitation during their hospitalisation had increased mortality (23.8\% versus $15.6 \%$; RR 1.54, 95\% CI 1.03-2.29), increased exercise capacity (mean difference $+107.92 \mathrm{~m}, 95 \% \mathrm{CI}+17.57 \mathrm{~m}$ to $+198.27 \mathrm{~m})$ and no difference in hospital readmissions $(52.9 \%$ versus 52.2\%; RR 0.88, 95\% CI 0.56-1.37), although all outcomes except mortality continued to have serious heterogeneity. The effect of pulmonary rehabilitation initiated after hospital discharge (up to 3 weeks after discharge) on mortality was uncertain due to the wide confidence interval ( $2.0 \%$ versus $7.8 \%$; RR 0.37, 95\% CI 0.06-2.29). However, pulmonary rehabilitation initiated after hospital discharge (up to 3 weeks after discharge) reduced hospital readmissions (21.5\% versus $46.8 \%$; RR $0.37,95 \%$ CI $0.14-0.97$ ) and improved quality of life (mean difference -11.75 , 95\% CI -19.76 to -3.75 ). Similarly, pulmonary rehabilitation initiated after hospital discharge (up to 8 weeks after discharge) increased exercise capacity (mean difference $+57.47 \mathrm{~m}, 95 \%$ CI $+20.04 \mathrm{~m}$ to $+94.89 \mathrm{~m}$ ). Again, all outcomes except mortality continued to have serious heterogeneity. The panel also hypothesised that differences in the way the pulmonary rehabilitation was conducted were responsible for the inconsistent results; however, this could not be tested. It is important to recognise, however, that the inconsistency across trials reflect variable magnitudes of effect (i.e. some studies showed a large benefit while others found a small benefit) and not differences in the direction of the effect.

Four of the trials evaluated adverse outcomes, three of which detected none [76, 78, 80]. The remaining trial reported that six (19\%) out of 32 patients had at least one adverse event (two events occurred in two patients in the control group, whereas 11 events occurred in four patients in the exercise groups) [88]. Only one of these adverse events was considered to be serious; a patient in one of the experimental groups had an episode of atrial fibrillation with accompanying chest pain.

\section{Benefits}

Pulmonary rehabilitation initiated during hospitalisation increased exercise capacity. Pulmonary rehabilitation initiated within 3 weeks following discharge reduced hospital readmissions and improved quality of life. Pulmonary rehabilitation initiated within 8 weeks following discharge increased exercise capacity.

\section{Harms}

Pulmonary rehabilitation initiated during hospitalisation increased mortality. Other serious adverse events occurring during pulmonary rehabilitation were rare. 


\begin{tabular}{|c|c|c|c|}
\hline & Recommendation & Strength & Quality of evidence \\
\hline 1 & $\begin{array}{l}\text { For ambulatory patients with an exacerbation of COPD, } \\
\text { we suggest a short course ( } \leqslant 14 \text { days) of oral corticosteroids }\end{array}$ & Conditional & Very low \\
\hline 2 & $\begin{array}{l}\text { For ambulatory patients with an exacerbation of COPD, } \\
\text { we suggest the administration of antibiotics }\end{array}$ & Conditional & Moderate \\
\hline 3 & $\begin{array}{l}\text { For patients who are hospitalised with a COPD exacerbation, } \\
\text { we suggest the administration of oral corticosteroids rather } \\
\text { than intravenous corticosteroids if gastrointestinal access } \\
\text { and function are intact }\end{array}$ & Conditional & Low \\
\hline 4 & $\begin{array}{l}\text { For patients who are hospitalised with a COPD exacerbation } \\
\text { associated with acute or acute-on-chronic respiratory failure, } \\
\text { we recommend the use of noninvasive mechanical ventilation }\end{array}$ & Strong & Low \\
\hline 5 & $\begin{array}{l}\text { For patients with a COPD exacerbation who present to the } \\
\text { emergency department or hospital, we suggest a home-based } \\
\text { management programme (hospital-at-home) }\end{array}$ & Conditional & Moderate \\
\hline 6 & $\begin{array}{l}\text { For patients who are hospitalised with a COPD exacerbation, } \\
\text { we suggest the initiation of pulmonary rehabilitation within } \\
3 \text { weeks after hospital discharge }\end{array}$ & Conditional & Very low \\
\hline 7 & $\begin{array}{l}\text { For patients who are hospitalised with a COPD exacerbation, } \\
\text { we suggest not initiating pulmonary rehabilitation during } \\
\text { hospitalisation }\end{array}$ & Conditional & Very low \\
\hline
\end{tabular}

\section{Other considerations}

The reliability of the estimated effects for all outcomes other than mortality is limited by inconsistency across trials in both the primary analysis and the stratified analysis. In addition to inconsistency, confidence in the estimated effects for all other outcomes was reduced because all of the trials had a risk of bias due to uncertain allocation concealment, lack of adherence to the intention-to-treat principle and/ or lack of blinding.

\section{Conclusions and research needs}

Pulmonary rehabilitation implemented during hospitalisation increases mortality. Pulmonary rehabilitation implemented within 3 weeks after discharge following a COPD exacerbation reduces hospital admissions and improves quality of life, while pulmonary rehabilitation implemented within 8 weeks after discharge increases exercise capacity. Research is needed to identify the interventions that provide the greatest benefits; some studies suggest that a combination of regular exercise with breathing technique training may be best, but additional investigations are needed. Studies employing methodologies of implementation science (also known as knowledge translation) are needed to test strategies that systematically target barriers and facilitators of integrating pulmonary rehabilitation into the care of patients with COPD exacerbations after hospital discharge.

What others are saying

The 2010 NICE guidelines concluded that "pulmonary rehabilitation should be made available to all appropriate people with COPD including those who have had a recent hospitalization for an acute exacerbation" [5].

\section{ERS/ATS recommendations}

For patients who are hospitalised with a COPD exacerbation, we suggest the initiation of pulmonary rehabilitation within 3 weeks after hospital discharge (conditional recommendation, very low quality of evidence).

For patients who are hospitalised with a COPD exacerbation, we suggest not initiating pulmonary rehabilitation during hospitalisation (conditional recommendation, very low quality of evidence).

\section{Remarks}

Early pulmonary rehabilitation refers to a programme that consists of physical exercise and education, which begins within 3 weeks of the start of treatment of the exacerbation. 
Values and preferences

This recommendation places a high value on improving clinical outcomes and a lower value on the burden and cost of pulmonary rehabilitation.

\section{Summary}

The Task Force utilised comprehensive evidence syntheses to inform its judgments regarding the balance of benefits versus burdens, adverse effects and costs; the quality of evidence; the feasibility; and the acceptability of various interventions for COPD exacerbations. A strong recommendation was made for NIV in patients with acute hypercapnic respiratory failure. Conditional recommendations were made for oral corticosteroids in outpatients, oral rather than intravenous corticosteroids in hospitalised patients, antibiotic therapy, home-based management of appropriately selected patients, and initiation of pulmonary rehabilitation within 3 weeks of hospital discharge (table 1). A conditional recommendation was made against the initiation of pulmonary rehabilitation during hospitalisation. The systematic review and GRADE methodology we employed for this ERS/ATS guideline indicated, in several instances, a sparse evidence base. In such cases, we recommend more definitive studies. These recommendations should be reconsidered as new evidence becomes available.

\section{References}

1 Seemungal TAR, Donaldson GC, Paul EA, et al. Effect of exacerbation on quality of life in patients with chronic obstructive pulmonary disease. Am J Respir Crit Care Med 1998; 157: 1418-1422.

2 Miravitlles M, Ferrer M, Pont A, et al. Effect of exacerbation on quality of life in patients with chronic obstructive pulmonary disease. A 2 year follow-up study. Thorax 2004; 59: 387-395.

3 Burrows B, Bloom JW, Traver GA, et al. The course and prognosis of different forms of chronic airways obstruction in a sample from the general population. N Engl J Med 1987; 317: 1309-1314

4 Donaldson GC, Seemungal TA, Bhowmilk A, et al. Relationship between exacerbation frequency and lung function decline in chronic obstructive pulmonary disease. Thorax 2002; 57: 847-852.

5 National Institute for Health and Clinical Excellence. Chronic obstructive pulmonary disease: management of chronic pulmonary obstructive disease in adults in primary and secondary care (partial update). London, National Clinical Guideline Centre, 2010.

6 Guyatt GH, Oxman AD, Kunz R, et al. GRADE guidelines: 2. Framing question and deciding on important outcomes. J Clin Epidemiol 2011; 64: 395-400.

7 National Institute for Health and Clinical Excellence. Chronic obstructive pulmonary disease: management of chronic pulmonary obstructive disease in adults in primary and secondary care (partial update). London, National Clinical Guideline Centre, 2004.

8 Schunemann HJ, Jaeschke R, Cook DJ, et al. An official ATS statement: grading the quality of evidence and strength of recommendations in ATS guidelines and recommendations. Am J Respir Crit Care Med 2006; 174: 605-614.

9 Jones PW, Beeh KM, Chapman KR, et al. Minimal clinically important differences in pharmacological trials. Am J Respir Crit Care Med 2014; 189: 250-255.

10 Andrews J, Guyatt G, Oxman AD, et al. GRADE guidelines: 14. Going from evidence to recommendations: the significance and presentation of recommendations. J Clin Epidemiol 2013; 66: 719-725.

11 Walters J, Gibson P, Wood-Baker R, et al. Systemic corticosteroids for acute exacerbations of chronic obstructive pulmonary disease. Cochrane Database Syst Rev 2009; 1: CD001288.

12 Quon BS, Gan WQ, Sin DD. Contemporary management of acute exacerbations of COPD: a systematic review and metaanalysis. Chest 2008; 133: 756-766.

13 Schweiger T, Zdanowicz M. Systemic corticosteroids in the treatment of acute exacerbations of chronic obstructive pulmonary disease. Am J Healt-Syst Pharm 2010; 167: 1061-1069.

14 Aaron SD, Vandemheen KL, Hebert P, et al. Outpatient oral prednisone after emergency treatment of chronic obstructive pulmonary disease. New Engl J Med 2003; 348: 2618-2625.

15 Thompson W, Nielson C, Carvalho P, et al. Controlled trial of oral prednisone in outpatients with acute COPD exacerbation. Am J Respir Crit Care Med 1996; 154: 407-412.

16 Bathoorn E, Liesker JJ, Postma DS, et al. Anti-inflammatory effects of combined budesonide/formoterol in COPD exacerbations. COPD J Chron Obstruct Pulm Dis 2008; 5: 282-290.

17 Bafadhel M, McKenna S, Terry S, et al. Blood eosinophils to direct corticosteroid treatment of exacerbations of chronic obstructive pulmonary disease. A randomized placebo-controlled trial. Am J Respir Crit Care 2012; 186: 48-55.

18 Bafadhel M, Davies L, Calverley PMA, et al. Blood eosinophil guided prednisolone therapy for exacerbations of COPD: a further analysis. Eur Respir J 2014; 44: 789-791.

19 Sayiner A, Aytemur ZA, Cirit M, et al. Systemic glucocorticoids in severe exacerbations of COPD. Chest 2001; 119: 726-730.

20 Leuppi JD, Schuetz P, Bingisser R, et al. Short-term vs conventional glucocorticoid therapy in acute exacerbations of chronic obstructive pulmonary disease. JAMA 2013; 309: 2223-2231.

21 Chen G, Xie CM, Luo YF. The effects and therapeutic duration of oral corticosteroids in patients with acute exacerbation of chronic obstructive pulmonary diseases. Chinese J Tuberc and Respir Dis 2008; 31: 577-580.

22 Global Initiative for Chronic Obstructive Lung Diseases. Global strategy for the diagnosis, management and prevention of chronic obstructive pulmonary disease (revised 2014). Global Inititative for Chronic Lung Disease, Inc., 2014.

23 Ram FS, Rodriguez-Roisin R, Granados-Navarrete A, et al. Antibiotics for exacerbations of chronic obstructive pulmonary disease. Cochrane Database Syst Rev 2006; 2: CD004403. 
24 Puhan MA, Vollenweider D, Latshang T, et al. Exacerbations of chronic obstructive pulmonary disease: when are antibiotics indicated? A systematic review. Respir Res 2008; 8: 30.

25 Vollenweider D, Jarrett H, Steurer-Stey C, et al. Antibiotics for exacerbations of chronic obstructive pulmonary disease. Cochrane Database Syst Rev 2012; 12: CD010257.

26 Anthonisen NR, Manfreda J, Warren C, et al. Antibiotic therapy in exacerbations of chronic obstructive pulmonary disease. Ann Intern Med 1987; 106: 196-204.

27 Jorgensen A, Coolidge J, Pedersen P, et al. Amoxicillin in treatment of acute uncomplicated exacerbations of chronic bronchitis. A double-blind, placebo-controlled multicentre study in general practice. Scand J Prim Health Care 1992; 10: 7-11.

28 Brusse-Keizer M, van der Valk P, Hendrix M, et al. Antibiotics in patients with a mild to moderate home-treated COPD exacerbation: the ABC trial. Am J Resp Crit Care Med 2009; 179: A1493.

29 Allegra L, Grossi E, Pozzi E, et al. The role of antibiotics in the treatment of chronic bronchitis exacerbation: follow up of a multicenter study. Italian J of Chest Dis 1991; 45: 138-148.

30 Llor C, Moragas A, Hernandez S, et al. Efficacy of antibiotic therapy for acute exacerbations of mild to moderate chronic obstructive pulmonary disease. Am J Respir Crit Care Med 2012; 186: 716-723.

31 Seemungal TA, Donaldson GC, Bhowmik A, et al. Time course and recovery of exacerbations in patients with chronic obstructive pulmonary disease. Am J Respir Crit Care Med 2000; 161: 1608-1613.

32 Miravitlles M, Moragas A, Hernández S, et al. Is it possible to identify exacerbations of mild to moderate COPD that do not require antibiotic treatment? Chest 2013; 144: 1571-1577.

33 de Jong YP, Uil SM, Grotjohan HP, et al. Oral or IV prednisolone in the treatment of COPD exacerbations: a randomized, controlled, double-blind study. Chest 2007; 132: 1741-1747.

34 Ceviker Y, Sayiner A. Comparison of two systemic steroid regimens for the treatment of COPD exacerbations. Pulm Pharmacol Ther 2014; 27: 179-183.

35 Krishnan JA, Mularski RA. Acting on comparative effectiveness research in COPD. JAMA 2010; 303: 2409-2410.

36 Lindenauer PK, Pekow PS, Lahit MC, et al. Association of corticosteroid dose and route of administration with risk of treatment failure in acute exacerbation of chronic obstructive pulmonary disease. JAMA 2010; 303: 2359-2367.

37 Ram FS, Picot J, Lightowler J, et al. Non-invasive positive pressure ventilation for treatment of respiratory failure due to exacerbations of chronic obstructive pulmonary disease. Cochrane Database Syst Rev 2004; 1: CD004104.

38 Andeev S, Tretyakov A, Grigoryants R, et al. Noninvasive positive airway pressure ventilation: role in treating acute respiratory failure caused by chronic obstructive pulmonary disease. Anesteziol Reanimatol 1998; 3: 45-51.

39 Barbe R, Togores B, Rubi M, et al. Noninvasive ventilatory support does not facilitate recovery from acute respiratory failure caused by chronic obstrucive pulmonary disease. Eur Respir J 1996; 9: 1240-1245.

40 Bott J, Carroll M, Conway J, et al. Randomised controlled trial of nasal ventilation in acute ventilatory failure due to chronic obstructive airways disease. Lancet 1993; 341: 1555-1557.

41 Brochard L, Mancebo J, Wysocki M, et al. Noninvasive ventilation for acute exacerbations of chronic obstructive pulmonary disease. New Engl J Med 1995; 333: 817-822.

42 Celikel T, Sungur M, Ceyhan B, et al. Comparison of nonivnasive positive pressure ventilation with standard medical therapy in hypercapnic acute respiratory failure. Chest 1998; 114: 1636-1642.

43 Conti G, Antonelli M, Navalesi P, et al. Non-invasive vs conventional mechanical ventilation in patients with chronic obstructive pulmonary disease after failure of medical treatment in the ward: a randomised trial. Intensive Care Med 2002; 28: 1701-1707.

44 del Castillo D, Barrot E, Laserna E, et al. Noninvasive positive pressure ventilation for acute respiratory failure in chronic obstructive pulmonary disease in a general respiratory ward. Med Clin (Barc) 2003; 120: 647-651.

45 Dikensoy O, Ikidag B, Filiz A, et al. Comparison of noninvasive ventilation and standard medical therapy in acute hypercapnic respiratory failure: a randomised controlled trial at a tertiary health centre in SE Turkey. Int J Clinical Pract 2002; 56: 85-88.

46 Khilnani GC, Saikia N, Banga A, et al. Non-invasive ventilation for acute exacerbation of COPD with very high $\mathrm{PaCO}_{2}$ : a randomized controlled trial. Lung India 2010; 27: 125-130.

47 Kramer N, Meyer T, Meharg J, et al. Randomised prospective trial of noninvasive positive pressure ventilation in acute respiratory failure. Am J Respir Crit Care Med 1995; 151: 1799-1806.

48 Plant P, Owen J, Elliott M. Early use of noninvasive ventilation for acute exacerbations of chronic obstructive pulmonary disease on general respiratory wards: a multicenter, randomised, controlled trial. Lancet 2000; 355: 1931-1935.

49 Servillo G, Ughi L, Rossano F, et al. Nonionvasive mask pressure support ventilation in COPD patients. Intensive Care Med 1994; 20: S54.

50 Thys F, Roeseler J, Reynaert M, et al. Noninvasive ventilation for acute respiratory failure: a prospective randomised placebo-controlled trial. Eur Respir J 2002; 20: 545-555.

51 Zhou $\mathrm{R}$, Chen $\mathrm{P}$, Luo $\mathrm{H}$, et al. Effects of noninvasive positive pressure ventilation on gas exchange and patients' transformation on chronic obstructive pulmonary disease and respiratory failure. Bull Hum Med Univ 2001; 26: 261-262.

52 Carrera M, Marin JM, Anton A, et al. A controlled trial of noninvasive ventilation for chronic obstructive pulmonary disease exacerbations. J Crit Care 2009; 24: 473.e7-14.

53 Keenan SP, Powers CE, McCormack DG. Noninvasive positive-pressure ventilation in patients with milder chronic obstructive pulmonary disease exacerbations: a randomized controlled trial. Respir Care 2005; 50: 610-616.

54 Pastaka C, Kostikas K, Karetsi E, et al. Non-invasive ventilation in chronic hypercapnic COPD patients with exacerbation and a pH of 7.35 or higher. Eur J Intern Med 2007; 18: 524-530.

55 Schmidbauer W, Ahlers O, Spies C, et al. Early prehospital use of non-invasive ventilation improves acute respiratory failure in acute exacerbation of chronic obstructive pulmonary disease. Emerg Med J 2011; 28: 626-627.

56 Vargas F, Bui HN, Boyer A, et al. Intrapulmonary percussive ventilation in acute exacerbations of COPD patients with mild respiratory acidosis: a randomized controlled trial. Crit Care 2005; 9: R382-R389.

57 Collaborative Research Group of Noninvasive Mechanical Ventilation for Chronic Obstructive Pulmonary Disease. Early use of non-invasive positive pressure ventilation for acute exacerbations of chronic obstructive pulmonary disease: a multicentre randomized controlled trial. Chin Med J 2005; 118: 2034-2040.

58 Dhamija A, Tyagi $\mathrm{P}$, Caroli $\mathrm{R}$, et al. Noninvasive ventilation in mild to moderate cases of respiratory failure due to acute exacerbation of chronic obstructive pulmonary disease. Saudi Med J 2005; 26: 887-890. 
59 Galli JA, Krahnke JS, James Mamary A, et al. Home non-invasive ventilation use following acute hypercapnic respiratory failure in COPD. Respir Med 2014; 108: 722-728.

60 Coughlin S, Liang WE, Parthasarathy S. Retrospective Assessment of Home Ventilation to Reduce Rehospitalization in Chronic Obstructive Pulmonary Disease. J Clin Sleep Med 2015; 11: 663-670.

61 Kohnlein T, Windisch W, Kohler D, et al. Non-invasive positive pressure ventilation for the treatment of severe stable chronic obstructive pulmonary disease: a prospective, multicentre, randomised, controlled clinical trial. Lancet Respir Med 2014; 2: 698-705.

62 Struik FM, Sprooten RT, Kerstjens HA, et al. Nocturnal non-invasive ventilation in COPD patients with prolonged hypercapnia after ventilatory support for acute respiratory failure: a randomised, controlled, parallelgroup study. Thorax 2014; 69: 826-834.

63 Jeppesen E, Brurberg K, Vist G, et al. Hospital at home for acute exacerbations of chronic obstructive pulmonary disease. Cochrane Database Syst Rev 2012; CD003573.

64 Cotton M, Bucknall C, Dagg K, et al. Early discharge for patients with exacerbations of chronic obstructive pulmonary disease: a randomised controlled trial. Thorax 2000; 55: 902-906.

65 Davies L, Wilkinson M, Bonner S, et al. 'Hospital at home' versus hospital care in patients with exacerbations of chronic obstructive pulmonary disease: prospective randomised controlled trial. BMJ 2000; 321: 1265-1268.

66 Hernandez C, Casas A, Escarrabill J, et al. Home hospitalisation of exacerbated chronic obstructive pulmonary disease patients. Eur Respir J 2003; 21: 58-67.

67 Nicholson C, Bowler S, Jackson C, et al. Cost comparison of hospital and home based treatment models for acute chronic obstructive pulmonary disease. Aust Health Rev 2001; 24: 181-187.

68 Nissen I, Jensen M. Nurse supported discharge of patients with exacerbation of chronic obstructive pulmonary disease. Ugeskr Laeger 2007; 169: 2220-2223.

69 Ojoo J, Moon T, McGlone S, et al. Patients' and carers' preferences in two models of care for acute exacerbations of COPD. Thorax 2002; 57: 167-169.

70 Ricuada N, Tibaldi V, Leff B, et al. Substitutive "hospital at home" versus inpatient care for elderly patients with exacerbations of chronic obstructive pulmonary disease: a prospective, randomised, controlled trial. J Am Geriatr Soc 2008; 56: 500.

71 Skwarska E, Cohen G, Skwarksi K, et al. Randomised controlled trial of supported discharge in patients with exacerbations of chronic obstructive pulmonary disease. Thorax 2000; 55: 907-912.

72 Utens C, Goossens L, Smeenk F, et al. Early assisted discharge with generic community nursing for chronic obstructive pulmonary disease exacerbations: results of a randomised controlled trial. BMJ Open 2012; 2: e001684.

73 Goosens L, Utens C, Smeenk F, et al. Cost-effectiveness of early assisted discharge for COPD exacerbations in the Netherlands. Value Health 2013; 16: 517-528.

74 Utens CMA, Goossens LMA, van Schayck OCP, et al. Patient preference and satisfaction in hospital-at-home and usual hospital care for COPD exacerbations: Results of a randomised controlled trial. Int J Nurs Stud 2013; 50: 1537-1549.

75 Puhan MA, Gimeno-Santos E, Scharplatz M, et al. Pulmonary rehabilitation following exacerbations of chronic obstructive pulmonary disease. Cochrane Database Syst Rev 2009; 1: CD005305.

76 Behnke M, Taube C, Kirsten D, et al. Home-based exercise is capable of preserving hospital-based improvements in severe chronic obstructive pulmonary disease. Respir Med 2000; 94: 1184-1191.

77 Carr SJ, Hill K, Brooks D, et al. Pulmonary rehabilitation after acute exacerbation of chronic obstructive pulmonary disease in patients who previously completed a pulmonary rehabilitation program. $J$ Cardiopulm Rehabil Prev 2009; 29: 318-324.

78 Eaton T, Young P, Fergusson W, et al. Does early pulmonary rehabilitation reduce acute health-care utilization in COPD patients admitted with an exacerbation? A randomized controlled study. Respirology 2009; 14: 230-238.

79 Kirsten DK, Taube C, Lehnigk B, et al. Exercise training improves recovery in patients with COPD after an acute exacerbation. Respir Med 1998; 92: 1191-1198.

80 Man WD, Polkey MI, Donaldson N, et al. Community pulmonary rehabilitation after hospitalisation for acute exacerbations of chronic obstructive pulmonary disease: randomised controlled study. BMJ 2004; 329: 1209.

81 Murphy N, Bell C, Costello RW. Extending a home from hospital care programme for COPD exacerbations to include pulmonary rehabilitation. Respir Med 2005; 99: 1297-1302.

82 Nava S. Rehabilitation of patients admitted to a respiratory intensive care unit. Arch Phys Med Rehabil 1998; 79: 849-854.

83 Seymour JM, Moore L, Jolley CJ, et al. Outpatient pulmonary rehabilitation following acute exacerbations of COPD. Thorax 2010; 65: 423-428.

84 Troosters T, Probst VS, Crul T, et al. Resistance training prevents deterioration in quadriceps muscle function during acute exacerbations of chronic obstructive pulmonary disease. Am J Respir Crit Care Med 2010; 181: 1072-1077.

85 Ghanem M, Elaal EA, Mehany M, et al. Home-based pulmonary rehabilitation program: effect on exercise tolerance and quality of life in chronic obstructive pulmonary disease patients. Ann Thorac Med 2010; 5: 18-25.

86 Ko FW, Dai DL, Ngai J, et al. Effect of early pulmonary rehabilitation on health care utilization and health status in patients hospitalized with acute exacerbations of COPD. Respirology 2011; 16: 617-624.

87 Deepak TH, Mohapatra PR, Janmeja AK, et al. Outcome of Pulmonary Rehabilitation in Patients after Acute Exacerbation of Chronic Obstructive Pulmonary Disease. Indian J Chest Dis Allied Sci 2014; 56: 7-12.

88 Tang CY, Blackstock FC, Clarence M, et al. Early rehabilitation exercise program for inpatients during acute exacerbation of chronic obstructive pulmonary disease: a randomized controlled trial. J Cardiopulm Rehabil Prev 2012; 32: 163-169.

89 Greening NJ, Williams JE, Hussain SF, et al. An early rehabilitation intervention to enhance recovery during hospital admission for an exacerbation of chronic respiratory disease: randomized controlled trial. BMJ 2014; 8: 349 . 